\title{
Coulomb Coupling between Spatially Separated Electron and Hole Layers: Generalized Random-Phase Approximation
}

\author{
H. C. Tso ${ }^{(a)}$ and P. Vasilopoulos \\ Department of Physics, Concordia University, 1455 de Maisonneuve Boulevard West, Montréal, Québec, Canada H3G 1 M8 \\ F. M. Peeters \\ Department of Physics, University of Antwerp (UIA), B-2610 Antwerp, Belgium
}

(Received 14 October 1992)

\begin{abstract}
Coulomb coupling between spatially separated quasi-two-dimensional electron and hole gases is studied as a function of temperature and/or electron (hole) gas density. Because of the exclusion principle mainly electrons and holes of antiparallel spin screen the electron-hole interaction at low densities. The coupling is described by a generalized random-phase approximation which takes into account exchange processes to all orders of the Hartree-Fock potential. The temperature dependence of the transimpedance agrees very well with experiment for relatively high densities; its density dependence agrees well for high densities and reasonably well for low and intermediate densities.
\end{abstract}

PACS numbers: $73.20 . \mathrm{Dx}$

Coulomb coupling between two spatially separated electron gases, when a current is driven through only one of them, has been predicted [1] and observed [2,3] to influence the transport properties of the individual gases. Recently, transport measurements have been reported [4] in a system composed of a two-dimensional (2D) electron gas (2DEG) and a 2D hole gas (2DHG). This system was proposed in Ref. [5] and its properties in a magnetic field are of strong current interest [6]. The gases are spatially separated by a barrier of width $d=200$ $\AA$. The barrier is high and thick enough to prevent tunneling and recombination but thin enough to allow for sizable Coulomb interaction between carriers in different gases. Current is allowed to flow in the electron gas and a drag voltage is developed and measured in the hole gas. The calculated [4] random-phase approximation (RPA) results for the temperature and density dependence of the coupling were a factor of 5 to an order of magnitude smaller than the experimental results for temperatures $(T)$ between $9 \mathrm{~K}$ and $50 \mathrm{~K}$. A similar discrepancy between theory and experiment [3] in an electron-electron system, for $T \leq 7 \mathrm{~K}$, has been explained, within $R P A$, by a phonon-mediated Coulomb coupling important only at these very low temperatures [7] and most pronounced for equal electron densities. This mechanism cannot explain the results of the electron-hole system since the temperatures of the experiment are high. Another mechanism would be electron-hole binding but this was estimated [4] to be very weak. So far there exists, to our knowledge, no explanation of these results. In this Letter, we show that at densities as low as $5 \times 10^{10} / \mathrm{cm}^{2}$ it is mainly electrons and holes of antiparallel spin that participate in the screening of the Coulomb interaction since a portion $[8,9]$ of them with parallel spins do not screen the Coulomb interaction due to the exclusion principle. Thus, the RPA treatment of the Coulomb scattering $[4,7]$ overestimates the screening and renders the coupling weaker. Using a generalized RPA (GRPA) approach, which takes into account exchange to all orders of the Hartree-Fock potential, we obtain a very good agreement with the experiment for the $T$ dependence of the transimpedance, a reasonable agreement for its density dependence at low densities, and a good one at high densities.

We model the electron-hole system with two infinitely deep quantum wells, of width $b$, with their centers and their closest edges separated by a distance $a$ and $d$, respectively. The many-body Hamiltonian describing this system is

$H(t)=H_{h}(t)+H_{e}(t)+H_{h e}(t)+H_{p}(t)+H_{i}(t)$.

Here, $H_{e}$ is the Hamiltonian of the electrons in the drive well and contains electron-electron, electron-impurity, and electron-phonon interactions; the same holds for the Hamiltonian of the holes in the drag well, $H_{h} . H_{h e}$ is the Coulomb interaction between holes and electrons in the two wells, $H_{p}$ is the phonon Hamiltonian, and $H_{i}$ the impurity Hamiltonian. For the densities of interest, only the lowest subband is occupied in either well. Equation (1) and the corresponding thermodynamic Green's function lead [7] to the coupled momentum-balance equations:

$$
\begin{aligned}
&-\frac{m_{e}^{*}}{e} \frac{\partial \mathbf{j}_{e}}{\partial t}+e n_{e} \mathbf{E}=\mathbf{F}^{e h}+\mathbf{F}^{e}, \\
& e n_{h} \frac{V_{D}}{L} \frac{\mathbf{j}_{h}}{j_{h}}+\frac{m_{h}^{*}}{e} \frac{\partial \mathbf{j}_{h}}{\partial t}=\mathbf{F}^{h e}+\mathbf{F}^{h} .
\end{aligned}
$$

Here, $V_{D}$ is the measured drag voltage, $L$ the length of the specimen, $n_{h}\left(n_{e}\right)$ the $2 \mathrm{D}$ hole density (electron density) of the drag (drive) well, $j_{h}\left(j_{e}\right)$ the $2 \mathrm{D}$ current density, $m_{e}^{*}\left(m_{h}^{*}\right)$ the electron (hole) effective mass, $\mathbf{E}$ the electric field applied only in the drive well, and $\mathbf{F}^{e}\left(\mathbf{F}^{h}\right)$ the frictional force due to phonon and impurity scattering with the electron (hole) gas. The term proportional to $V_{D}$ develops when the current is not allowed to flow 
in the hole well; if it is allowed to flow, this term vanishes identically. Ignoring the vertex corrections of the three-point vertex function [see Eq. (149) of Ref. [10]], the self-energy of the holes is related to the nonequilibrium screened interaction $\tilde{v}_{h e}$ and the nonequilibrium hole Green's function as illustrated in Fig. 1(a). The total force acting on the holes is

$\mathbf{F}^{h e}+\mathbf{F}^{h}=\int d z_{1} \int_{t_{0}}^{t_{1}} d(3)\left[\Pi_{>}^{h} \nabla_{1} \tilde{v}_{h e>}-\Pi_{<}^{h} \nabla_{1} \tilde{v}_{h e<}\right]$

where $\Pi_{\gtrless}^{h} \equiv \Pi_{\gtrless}^{h}(1 ; 3)\left(\Pi_{\gtrless}^{e}\right)$ is the nonequilibrium hole (electron) polarizability, and $\tilde{v}_{h e} \equiv \tilde{v}_{h e}(1 ; 3)$ is the nonequilibrium screened interaction between holes in the drag well and the medium (electrons in the drive well, phonons, and impurities included). The arguments in $\Pi^{h}(1 ; 2)$ and $\tilde{v}_{h e}(1 ; 2)$ represent the correlation between the space-time coordinates $\left(\mathbf{x}_{1}, t_{1}\right)$ and $\left(\mathbf{x}_{2}, t_{2}\right)$ and $\int_{t_{0}}^{t_{1}} d(3)$ is a shorthand for $\int d \mathbf{x}_{3} \int_{t_{0}}^{t_{1}} d t_{3}$. The coupling force due to Coulomb scattering $\mathbf{F}^{\text {he }}$ is given within RPA by

$\mathbf{F}^{h e}=\int_{t_{0}}^{t_{1}} d(5) \int_{-\infty}^{\infty} d(4) \int_{-\infty}^{\infty} d(3)\left[\bar{v}_{+}(1 ; 3) \Pi_{>}^{e}(3 ; 4) \nabla_{1}^{T} \bar{v}_{-}(4 ; 5) \Pi_{<}^{h}(5 ; 1)-\Pi_{<}^{h}(1 ; 5) \nabla_{1} \bar{v}_{+}(1 ; 3) \Pi_{<}^{e}(4 ; 3) \bar{v}_{-}(4 ; 5)\right]$
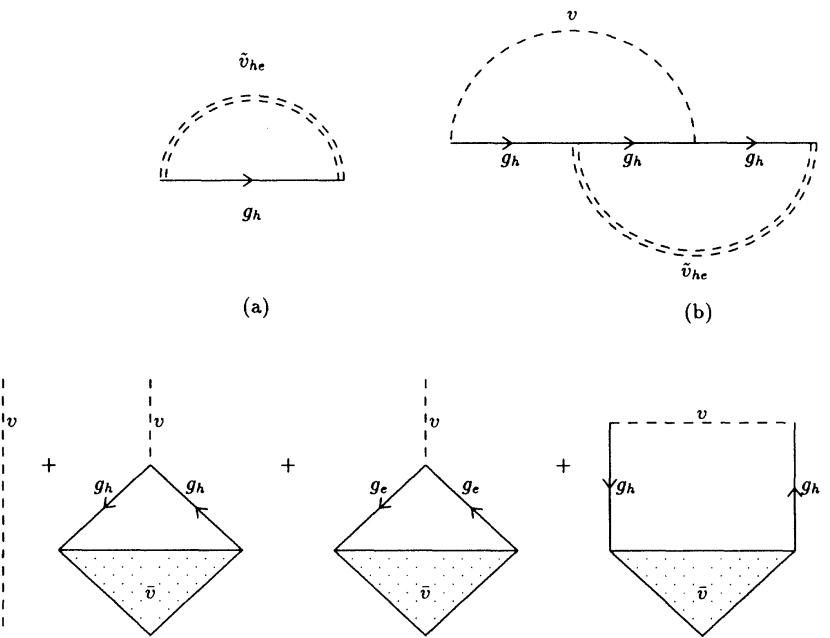

(a)

(b)

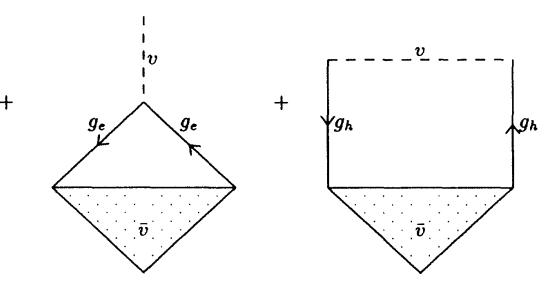

(c)

FIG. 1. (a) Self-energy diagram of holes in RPA; (b) additional self-energy diagram of holes due to the exchange process; (c) self-consistent screened potential in GRPA.

with $\bar{v}_{h e \pm} \equiv \bar{v}_{h e \pm}(1 ; 3)$ the retarded (advanced) screened Coulomb interaction between holes in the drag well and the electrons in the drive well, $\nabla_{1}^{T}$ standing for $\nabla_{1}$ operating to the left. The Fourier component of the screened Coulomb interaction can be written as $\bar{v}_{ \pm}\left(\mathbf{q}_{\|} ; \omega\right)=F\left(q_{\|}\right) v\left(q_{\|} ; a\right) / \epsilon_{ \pm}\left(\mathbf{q}_{\|} ; \omega\right)$, where $v\left(q_{\|} ; a\right)=2 \pi e^{2} e^{-q_{\|} a} / q_{\|}$, with $\zeta=q_{\|} b$ and $F\left(q_{\|}\right)$the form factor [7]. The retarded (advanced) dielectric function $\epsilon_{ \pm}(\cdots)$ is given by

$$
\begin{aligned}
\epsilon_{ \pm}\left(\mathbf{q}_{\|} ; \omega\right)= & {\left[1-v\left(q_{\|} ; 0\right) F\left(q_{\|}\right) \Pi_{ \pm}^{h(0)}\left(\mathbf{q}_{\|} ; \omega\right)\right]\left[1-v\left(q_{\|} ; 0\right) F\left(q_{\|}\right) \Pi_{ \pm}^{e(0)}\left(\mathbf{q}_{\|} ; \omega\right)\right] } \\
& -\left|v\left(q_{\|} ; a\right) F\left(q_{\|}\right)\right|^{2} \Pi_{ \pm}^{h(0)}\left(\mathbf{q}_{\|} ; \omega\right) \Pi_{ \pm}^{e(0)}\left(\mathbf{q}_{\|} ; \omega\right),
\end{aligned}
$$

where $\Pi^{h(0)}\left(\Pi^{e(0)}\right)$ is the equilibrium hole (electron) polarizability.

For weak electric fields, we may write $\mathbf{j}_{e, h}=n_{e, h} p e \mathbf{v}_{d}^{e, h}$ with $\mathbf{v}_{d}^{e}\left(\mathbf{v}_{d}^{h}\right)$ the drift velocities, $p=1$ for electrons, and $p=-1$ for holes. Then the forces can be linearized over $\mathbf{v}_{d}^{e}$ and $\mathbf{v}_{d}^{h}$ giving $\mathbf{F}^{h}=\left(\Omega_{h i}+\Omega_{h p}\right) m_{h} \mathbf{v}_{d}^{h}$ and $\mathbf{F}^{h e}=\Omega_{h e} m_{h}\left(\mathbf{v}_{d}^{h}-\mathbf{v}_{d}^{e}\right)$. Here $\Omega_{h e}, \Omega_{h p}$, and $\Omega_{h i}$ are the relaxation frequencies per unit area due to the Coulomb coupling, phonon scattering, and impurity scattering, respectively. In the steady state and in the absence of the drag current $\left(j_{h}=0\right)$, Eq. (5) gives the transimpedance $R_{T} \equiv V_{D} / I_{e}=m_{h} \Omega_{h e} / e^{2} n_{h} n_{e}$ and the frequency

$$
\Omega_{h e}=-\left.2 \hbar \int_{-\infty}^{\infty} \frac{d \omega}{2 \pi} \sum_{\mathbf{q}_{\|}} \frac{\left|F\left(q_{\|}\right) v\left(q_{\|} ; a\right)\right|^{2}}{\left|\epsilon_{+}\left(\mathbf{q}_{\|} ; \omega\right)\right|^{2}} q_{\|}^{2} \frac{\partial n\left(\omega^{\prime}\right)}{\partial \omega^{\prime}}\right|_{\omega^{\prime}=-\omega} \operatorname{Im}\left[\Pi_{+}^{h(0)}\right] \operatorname{Im}\left[\Pi_{+}^{e(0)}\right],
$$

with $\Pi_{+}^{h(0)} \equiv \Pi_{+}^{h(0)}\left(\mathbf{q}_{\|} ; \omega\right)$. For $T>10 \mathrm{~K}, \Omega_{h e}$ behaves approximately linearly with $T$ as shown by the dashed curve in Fig. 4. As is demonstrated in Figs. 2, 3, and 4, and also in Ref. [4], there is a marked difference between the RPA calculations and the experimental results. This difference tends to be larger at lower densities as illustrated in Figs. 2 and 3. This is mainly due to the fact that RPA is good $[8,9]$ only for very high densities. An extra term [10], as shown in Fig. 1(b), should be included in the self-energy for lower densities. This term takes into account the exchange process of holes and electrons. It can be ignored for high densities but not for low densities as shown by Abrikosov, Gorkov, and Dzyaloshinski [11]. With this extra term, the screened Coulomb interaction $\bar{v}(1 ; 2)$ is no longer a two-point but a three-point interaction $\bar{v}(1,2 ; 3)$ as expressed diagramatically in Fig. 1(c). The diagrams in Fig. 1(c) represent the right-hand side (RHS) of an equation whose left-hand side is equal to the shaded triangle that represents $\bar{v}$. Thus $\bar{v}$ obeys an integral equation. Within RPA the last diagram in Fig. 1 (c) is absent. Solving this equation is equivalent to solving the equation of motion for the density operator to all 


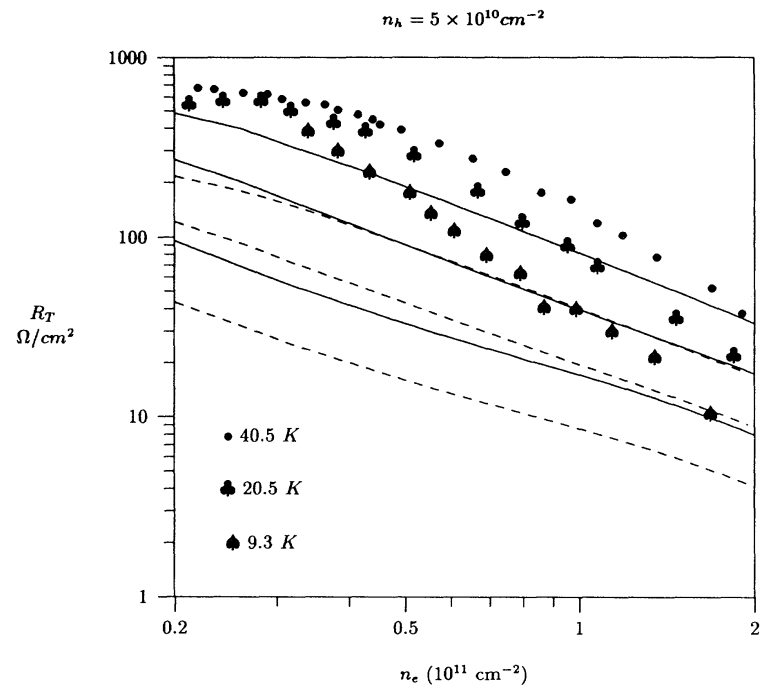

FIG. 2. Transimpedance $R_{T}$ per square vs electron density $n_{e}$ for three temperatures, with fixed hole density $n_{h}=$ $5 \times 10^{10} / \mathrm{cm}^{2}$. The solid and dashed curves are the GRPA and RPA results, respectively. The solid dots are the experimental results of Ref. [4].

orders in the Hartree-Fock potential as done by Nozières and Pines [8]. However, we need to know the nonequilibrium polarizabilities. To simplify the problem, we do an iteration in the coupling force $\mathbf{F}^{\text {he }}$ by letting $\mathbf{F}^{\text {he }} \rightarrow 0$ (the polarizabilities depend on this force) and find out the relationship between the nonequilibrium $\Pi^{h}$ and $\Pi^{e}$. Then we use this relation to solve for $\bar{v}$. This procedure is good only for the steady state and when phonon and impurity scatterings are weak. This becomes obvious if we let the current flow in the hole system. Once the

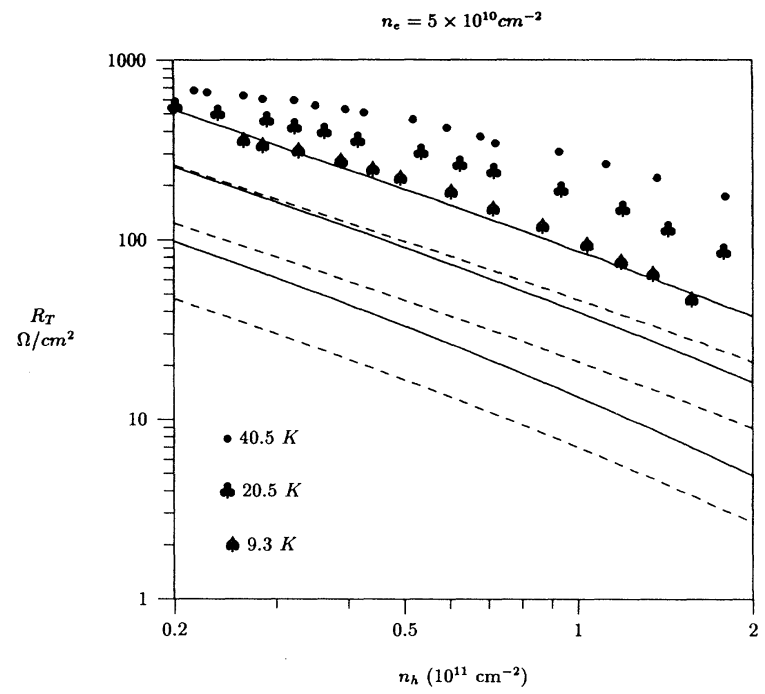

FIG. 3. $R_{T}$ per square vs hole density for three temperatures with fixed electron density $n_{e}=5 \times 10^{10} / \mathrm{cm}^{2}$. All curves are marked as in Fig. 2.

system reaches a steady state, the momentum transfer from the electrons should be balanced by phonon and impurity scattering. If both phonon and impurity scatterings are weak, $\mathbf{F}^{\text {he }} \simeq 0$. With this condition, Eq. (5) gives $\Pi^{h} \simeq \Pi^{e}$. Further, to reduce the numerical work, we assume, though the temperature is not too low, that the main scattering occurs near the electron and hole Fermi levels so that $\bar{v}(1,2 ; 3) \simeq \bar{v}(1 ; 3) \delta(1 ; 2)$. With these approximations, we solve the equation for $\bar{v}$ and obtain $\bar{v}_{ \pm}\left(\mathbf{q}_{\|} ; \omega\right)=v(q ; a) / \epsilon_{ \pm}^{G}\left(\mathbf{q}_{\|} ; \omega\right)$, where the GRPA dielectric function $\epsilon_{ \pm}^{G}(\cdots)$ is

$$
\begin{aligned}
\epsilon_{ \pm}^{G}\left(\mathbf{q}_{\|} ; \omega\right) \simeq\left[1-v_{\mathrm{HF}}^{h}\left(q_{\|} ; 0\right) \Pi_{ \pm}^{h(0)}\left(\mathbf{q}_{\|} ; \omega\right)\right]\left[1-v_{\mathrm{HF}}^{e}\left(q_{\|} ; 0\right) \Pi_{ \pm}^{e(0)}\left(\mathbf{q}_{\|} ; \omega\right)\right] \\
-v_{\mathrm{HF}}^{h}\left(q_{\|} ; a\right) v_{\mathrm{HF}}^{e}\left(q_{\|} ; a\right) e^{-2 q_{\|} a} \Pi_{ \pm}^{h(0)}\left(\mathbf{q}_{\|} ; \omega\right) \Pi_{ \pm}^{e(0)}\left(\mathbf{q}_{\|} ; \omega\right),
\end{aligned}
$$

with

$$
v_{\mathrm{HF}}^{h, e}\left(q_{\|} ; a\right)=\frac{2 \pi e^{2}}{q_{\|}}\left[F\left(q_{\|}\right)-\frac{1}{4} \frac{q_{\|}}{k_{F}^{h, e}+q_{\|}} F\left(q_{\|}+k_{F}^{h, e}\right) e^{-k_{F}^{h, e} a}\right],
$$

The second term on the RHS of Eq. (9) is the exchange term and occurs only for electrons (or holes) of parallel [8] spin. As a result, only electrons (or holes) of antiparallel spin and a portion of electrons (or holes) of parallel spin will participate, statistically speaking, in the screening for $q_{\|} \gg k_{F}$, i.e., for low densities. Physically, this means that a portion [depending on $q_{\|}$in Eq. (9)] of electrons (or holes) of the same spin will be kept apart by the Pauli exclusion principle. For very low densities, they can be so far apart that they have almost no effect on the screening at all. If carriers in both wells are of the same type, we will have a factor of $1 / 2$ in the exchange
[8] term instead of $1 / 4$. This happens because there is no exchange process between an electron and a hole. With the obtained $\bar{v}, \Omega_{h e}$ takes the form of Eq. (7) with $\epsilon_{ \pm}(\cdots)$ replaced by $\epsilon_{ \pm}^{G}(\cdots)$.

The calculated GRPA and RPA results for the transimpedance $R_{T}$ are shown in Figs. 2-4 by the solid and dashed curves, respectively. In Fig. $2 R_{T}$ is shown as a function of electron density $n_{e}$ for fixed hole density $n_{h}$ and in Fig. 3 as a function of $n_{h}$ for fixed $n_{e}$. The solid symbols are the experimental results of Ref. [4]. We have used $m_{h}^{*}=6.7 m_{e}^{*}$ and the parameters of the experiment. Noticing the logarithmic scale in Figs. 2 and 3 it 


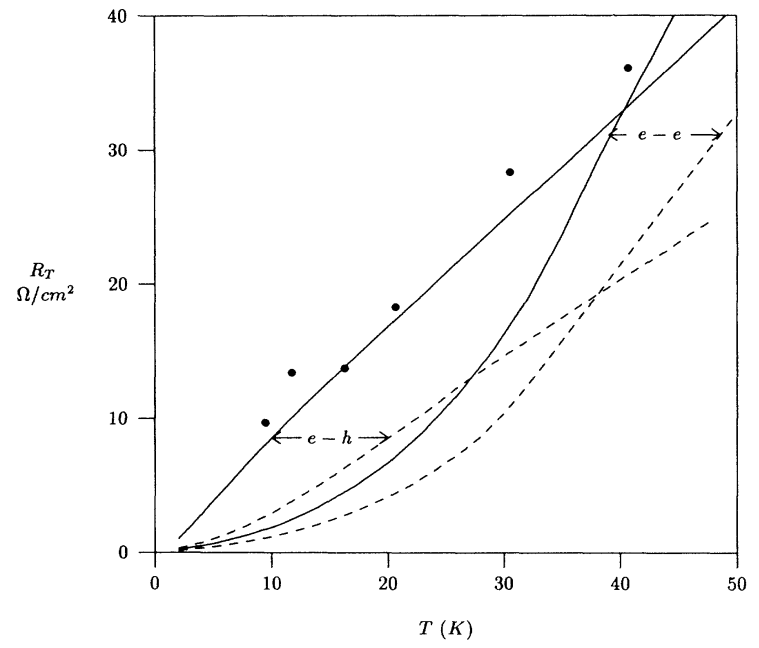

FIG. 4. $R_{T}$ per square vs temperature for fixed densities. Curves $e-h: n_{e}=2 \times 10^{11} / \mathrm{cm}^{2} ; n_{h}=5 \times 10^{10} / \mathrm{cm}^{2}$. Curves $e-e:$ in both wells $n_{e}=1.5 \times 10^{11} / \mathrm{cm}^{2}$. All curves are marked as in Fig. 2.

is seen that the density dependence of the GRPA $R_{T}$ is (i) significantly better than the RPA result, (ii) reasonably good at low densities, (iii) very good at relatively high electron densities, and (iv) reasonably good for high hole densities. For intermediate densities the GRPA result is smaller than the experimental one. The better agreement at high densities is due to the approximation $\bar{v}(1,2,3) \approx \bar{v}(1 ; 3) \delta(1,2)$ that becomes increasingly better as the density increases and the gases become more degenerate. On the other hand, the temperature dependence of the GRPA $R_{T}$ (solid $e-h$ curves), at relatively high densities, shown in Fig. 4, is in very good agreement with the experimental result. For contrast we also show $R_{T}$ for the same structure with electrons in both wells ( $e-e$ curves) and equal densities $n_{e}=1.5 \times 10^{11} / \mathrm{cm}^{2}$. The difference between the GRPA and RPA results is less pronounced than in the electron-hole system at low temperatures mainly because both electron densities are rather high since, as we have verified, the effective-mass dependence of $R_{T}$ is very weak.

At low to moderate densities our GRPA results show a stronger $T$ dependence than the experimental ones. A possible explanation is that at the high temperatures of the experiment the particle wave function spreads due to the increase in the kinetic energy which acts against the confining potential or electric field $E_{l}, l=e, h$. This corresponds to effectively temperature dependent well widths. Equating the pressure exerted by the gases [12] $P_{l}=n_{l}^{3 D} k_{B} T \alpha_{l}$ with that by the field $E_{l}$ we obtain $d_{l}=d_{l 0}+\alpha_{l} k_{B} T / e E_{l}$, where $d_{l 0}$ is the $T=0$ width. In GRPA $\alpha_{l}=1-(3 / 72) \pi r_{D}^{3} n_{l}^{3 D}+B\left(n_{l}^{3 D}\right)$ and $B(\cdots)$ is a positive function that vanishes at high densities. Thus $\alpha_{l}$ and $d_{l}$ increase at low densities and so does the mean separation $a$ between electrons and holes. This leads to a decrease in $R_{T}$ approximately compensated by the increase shown in Figs. 2, 3, and 4.

In all cases the RPA results are significantly below the experimental or GRPA results since RPA overestimates screening. Also, they differ from those of the experiment by a factor of 2 rather than a factor of 5 as reported by Sivan, Solomon, and Shtrikman [4]. This is because the quantum wells have finite thickness $\left[F\left(q_{\|}\right)<1\right]$ in our case and zero $\left[F\left(q_{\|}\right)=1\right]$ in theirs.

In summary, we have shown that the reported [4] deviations of density and temperature dependences of the Coulomb coupling between an electron gas and a hole gas, spatially separated from each other, from the RPA results are mostly due to an overestimation of the screening when treated within RPA. Most of the results, especially the temperature dependence, can be explained by employing a GRPA which takes into account exchange processes, absent from RPA, in the two wells to all orders of the Hartree-Fock potential.

This work was supported by NSERC Grant No. OGPIN028 (H.C.T. and P.V.) and by the Collaborative Research Grant NATO: 5-2-05/RG No. 890123 (P.V., F.M.P.).

(a) Present address: Department of Physics, Queen's University, Kingston, Canada K7L 3N6.

[1] M. B. Pogrebinskii, Fiz. Tekh. Poluprovodn. 11, 637 (1977) [Sov. Phys. Semicond. 11, 372 (1977)]; P. J. Price, Physica (Amsterdam) 117B, 750 (1983); I. I. Boiko and Yu. M. Sirenko, Phys. Status Solidi 159, 805 (1990).

[2] P. M. Solomon, P. J. Price, D. J. Frank, and D. C. La Tulipe, Phys. Rev. Lett. 63, 2508 (1989).

[3] T. J. Gramila, J. Eisenstein, A. H. MacDonald, L. N. Pfeiffer, and K. W. West, Phys. Rev. Lett. 66, 1216 (1991).

[4] U. Sivan, P. M. Solomon, and H. Shtrikman, Phys. Rev. Lett. 68, 1196 (1992).

[5] Yu. E. Lozovik and V. I. Yudson, Pis'ma Zh. Eksp. Teor. Fiz. 22, 556 (1975) [JETP Lett. 22, 274 (1975)].

[6] X. M. Chen and J. J. Quinn, Phys. Rev. Lett. 67, 895 (1991).

[7] H. C. Tso, P. Vasilopoulos, and F. M. Peeters, Phys. Rev. Lett. 68, 2516 (1992).

[8] P. Nozières and D. Pines, Phys. Rev. 111, 442 (1958).

[9] J. Hubbard, Proc. R. Soc. London A 243, 336 (1957).

[10] H. C. Tso and N. J. M. Horing, Phys. Rev. B 44, 8886 (1991).

[11] A. A. Abrikosov, L. P. Gorkov, and I. E. Dzyaloshinski, Methods of Quantum Field Theory in Statistical Physics (Dover, New York, 1963), p. 336.

[12] L. P. Kadanoff and G. Baym, Quantum Statistical Mechanics (Benjamin, New York, 1962), p. 175. 